FINEZ Jean et COMET Catherine (2011), «Solidarités patronales et formation des interlocks entre les principaux administrateurs du CAC40 », Terrains \& Travaux, $\mathbf{n}^{\circ} 19$, p. 57-76.

\author{
Jean Finez \\ Doctorant contractuel en sociologie \\ CLERSÉ - UMR CNRS 8019 \\ Université de Lille 1 \\ Bâtiment SH1 - Bureau 15 \\ 59655 Villeneuve d'Ascq Cedex
}

Courriel : jean.finez@ed.univ-lille1.fr

Tél. : 0320336299

\title{
Catherine Comet
}

Maître de conférences en sociologie

CLERSÉ - UMR CNRS 8019

Université de Lille 1

Bâtiment SH2 - Bureau 122

59655 Villeneuve d'Ascq Cedex

Courriel : catherine.comet@univ-lille1.fr

Tél. : 0320434687

\section{Solidarités patronales et formation des interlocks entre les principaux administrateurs du CAC40}

Résumé : Du fait des cumuls de mandats, les entrecroisements entre les conseils d'administration (en anglais, interlocks) des sociétés du CAC40 forment un réseau particulièrement dense. Les intercooptations reflètent des solidarités dont nous cherchons à élucider quelques-uns des fondements en nous focalisant sur les dirigeants qui cumulent au moins trois sièges dans les organes de gouvernance des sociétés du CAC40. Si les solidarités de corps liées au passage par les mêmes grandes écoles - essentiellement Polytechnique et l'ENA - éclairent une part de ces liens, il apparaît que les affinités idéologiques voire les

relations d'amitié, mesurées à partir des coappartenances dans des clubs ou think tanks libéraux, jouent également un rôle. 


\title{
Solidarity and interlocks formation among the main CAC40 directors
}

\begin{abstract}
Because of multiple directorships, the overlaps among the boards of CAC40 companies form a particularly dense network. Focusing on the directors who seat in more than two boards of CAC4O companies, we aim to emphasize the underlying bases of the solidarity, which trigger intercooptations. If the solidarity based upon an education provided by the same elite school, essentially Polytechnique and ENA, sheds light on a part of these ties, ideological affinities and friendships, measured by common memberships in clubs and liberal thinks tanks, also play a significant role.
\end{abstract}




\section{Solidarités patronales et formation des interlocks entre les principaux administrateurs du CAC40}

En France, les élites patronales n'ont jamais eu très bonne réputation ${ }^{1}$. On reproche notamment à leurs membres de se coopter les uns les autres dans les conseils d'administration des grandes sociétés qu'ils dirigent, conférant au patronat français un aspect «consanguin ». Les «grands patrons» se défendent de telles pratiques et affirment qu'elles seraient aujourd'hui révolues. Pierre Bilger - ancien président-directeur général d'Alstom - déclare par exemple en 2007 : «en harmonie avec les exigences du gouvernement d'entreprise, de telles situations [les pratiques d'intercooptation] qui n'étaient pas exceptionnelles dans le passé, sont aujourd'hui, à juste titre, devenues extrêmement rares, pour ne pas dire inexistantes, au moins dans les grandes sociétés cotées. De même, le nombre de postes effectivement détenus par un administrateur s'est considérablement réduit. » ${ }^{2}$

Les propos de l'ancien dirigeant sont cependant en contradiction avec nos observations. Notre article vise à éclairer les solidarités mobilisées au sein du grand patronat, et plus précisément les formes de solidarité limitée (Granovetter, 1995 ; Lazega, 1999). D’un point de vue méthodologique, nous nous interrogeons sur les apports et les enjeux de l'analyse de réseaux dans le cadre d'un renouvellement des recherches sur le patronat et nous montrons en quoi l'utilisation de cette approche permet d'effectuer un va-et-vient entre la mobilisation de matériaux qualitatifs et un traitement quantitatif de données relationnelles.

Nous faisons l'hypothèse que les dirigeants échangent des sièges d'administrateurs avec des partenaires privilégiés en fonction de certaines ressemblances. Ces critères identitaires facilitent les échanges car ils préjugent de valeurs et d'intérêts communs. Notre démarche consiste à repérer des sous-groupes cohésifs fondés sur des propriétés ou affiliations communes, témoignant de cette solidarité limitée. Nous analysons cette question

\footnotetext{
$1 \quad$ Cet article est issu d'un mémoire de Master 2 de sociologie réalisé par Jean Finez en 2009, sous la direction de Catherine Comet. Ayant fait l'objet d'une communication aux journées d'études «Élites économiques en France et en Europe » (Univ. Paris-Dauphine, 4-5 novembre 2010), il a bénéficié des nombreuses remarques et suggestions des participants que nous remercions ici. Nous remercions également les relecteurs anonymes, ainsi que les coordinatrices du numéro et le comité de rédaction de Terrain \& Travaux, dont le travail a permis d'améliorer substantiellement la qualité de notre article.

2 Pierre Bilger, Causeries à bâtons rompus. Bloc-notes, Le Publieur, Paris, 2007, p. 70.
} 
en nous intéressant aux principaux administrateurs des entreprises du CAC40 et aux interlocking directorships ou interlocks, c'est-à-dire aux liens de coappartenance dans les conseils d'administration ${ }^{3}$. Conformément à la littérature sociologique, d'après laquelle les formations scolaires structurent l'univers des élites françaises (Suleiman, 1979; Bourdieu, 1989 ; Bellier, 1992), nous cherchons à déterminer dans quelle mesure l'homophilie scolaire permet de comprendre l'existence des liens interlocks entre les dirigeants des sociétés du CAC40. L'explication par les profils scolaires communs se révèle féconde mais limitée. La prise en compte des liens d'affinité idéologique voire d'amitié entre dirigeants améliore la compréhension du réseau interlocks.

\section{Encadré 1 : Le cadre institutionnel régulant le cumul de mandat}

Plusieurs textes législatifs encadrent la gouvernance des sociétés de droit français. Ainsi, jusqu'à 18 membres peuvent siéger dans un conseil d'administration ou un conseil de surveillance d'une société anonyme. Des préconisations de bonnes pratiques invitent même les très grandes entreprises françaises à réduire la taille des conseils. En outre, la loi de 2001 relative aux nouvelles régulations économiques - dite «loi NRE » - fixe à 5 le nombre de mandats, toutes natures confondues (directeur général, membre du directoire, administrateur ou membre du conseil de surveillance), qu'une même personne physique peut exercer simultanément en France. Ce faisant, elle a entraîné de nombreuses démissions. En 2006, tous les dirigeants du CAC40 respectent ce point de la loi NRE, ce qui signifie qu'ils ont opéré des choix concernant les instances dirigeantes dans lesquelles ils souhaitaient continuer à siéger. Dès lors, la présence dans un conseil d'administration a, selon nous, une signification sociale renforcée.

\section{1. Étudier les réseaux des dirigeants}

\section{L'analyse de réseaux, un complément aux approches typologiques}

Les travaux sociologiques sur les dirigeants d'entreprise se concentrent souvent sur leurs attributs individuels (trajectoires scolaires, carrières professionnelles ou encore origines sociales). Les recherches les plus abouties qui continuent à faire référence aujourd'hui sont, d'une part les travaux critiques de Pierre Bourdieu (voir notamment Bourdieu et Saint Martin, 1978 ; Bourdieu, 1989), d'autre part ceux de Michel Bauer et Bénédicte Bertin-Mourot (Bauer, 1988 ; Bauer et Bertin-Mourot, 1987). ${ }^{4}$ L'objectif de ces travaux est de proposer une typologie des dirigeants afin de comprendre les logiques, les tensions et les rapports de force de l'univers social considéré. Qu'ils soient divisés en deux groupes - «patrons privés » et «patrons d'État» (Bourdieu et Saint Martin, 1978) -, ou en trois groupes - «patrons propriétaires », «princes du diplôme» et «dirigeants maisons » (Bauer, 1988) -, les

\footnotetext{
On dit qu'il existe un lien interlock entre les dirigeants $\mathrm{A}$ et $\mathrm{B}$, dès lors qu'ils sont simultanément présents au sein des instances dirigeantes d'une même entreprise (voir Mizruchi, 1996).

Il existe d'autres recherches sur lesquelles nous ne revenons pas ici. Cela ne doit préjuger en rien de l'intérêt que nous portons à ces travaux : $c f$. par exemple les recherches de Michel Villette et Catherine Vuillermot (2005) qui portent sur la trajectoire de quelques dizaines d' «hommes d'affaires » de carrure internationale.
} 
dirigeants d'entreprise sont définis à partir de leurs attributs individuels, et les liens sociaux qu'ils entretiennent les uns avec les autres sont peu pris en compte.

Bauer et Bertin-Mourot (1987) soulignent, à juste titre, l'importance des relations et des réseaux pour comprendre les logiques d'accès aux plus hauts sommets de l'entreprise, mais cette perspective ne constitue pas un aspect essentiel de leur enquête. P. Bourdieu a élaboré un cadre théorique pour l'étude des relations sociales à travers le concept de capital social (Bourdieu, 1980). Néanmoins, la définition qu'il en donne reste difficile à opérationnaliser, même si elle cristallise selon nous la volonté de combiner une analyse en termes de champ avec une étude des réseaux relationnels.

L'analyse du «champ patronal » (et, d'une manière plus large, des champs sociaux dans toute leur diversité, au moins depuis l'ouvrage La distinction) repose sur le traitement statistique d'informations biographiques (Bourdieu et Saint Martin, 1978). L'image de l'espace social est en ce sens représentée grâce à une technique d'analyse géométrique des données : l'analyse des correspondances multiples. Cette méthode a pour principale qualité de permettre de figurer spatialement les rapports de force au sein du champ en rapprochant les individus (ou les variables) qui sont «homogènes » (c'est-à-dire positivement corrélées). Mais des profils homologues, et donc proches spatialement, ne signifient pas que les individus se connaissent et se fréquentent. Seule une véritable analyse des réseaux sociaux permet d'étudier l'existence, la nature et la force des relations entre les agents sociaux. Pour compléter l'analyse de la «noblesse d'État» (Bourdieu, 1989) et ses réactualisations (Dudouet et Grémont, 2007), nous proposons de cartographier une forme de relations sociales - en l'occurrence les interlocks - entre des dirigeants de grandes entreprises françaises, et d'éclairer ces liens à partir de certains de leurs attributs et affiliations. Par ailleurs, l'un des principaux intérêts de l'analyse de réseaux sociaux, par rapport à l'analyse factorielle, est de mettre au jour l'existence de processus sociaux, reposant sur des interdépendances en ressources.

\section{Comment comprendre la formation des interlocks?}

En France, plusieurs sociologues ont analysé les liens interlocks au sein du patronat. Deux recherches ont particulièrement inspiré notre étude. Au début des années 1990, Charles Kadushin a réalisé une enquête, avec l'aide d'une journaliste française, sur les liens d'amitié et l'existence de liens interlocks entre 28 dirigeants appartenant à l'élite financière française. Il montre que le passage par l'ENA, le fait d'avoir la même origine sociale ou encore de partager des mêmes sensibilités politiques favorise l'existence de liens d'amitié entre dirigeants et, dans une moindre mesure, la coprésence dans les mêmes conseils d'administration (Kadushin, 1995). Dans une enquête portant sur un tout autre terrain mais utilisant les techniques de l'analyse de réseaux, Pierre-Paul Zalio s'est s'intéressé aux liens interlocks au sein de la bourgeoisie marseillaise des années 1930 (Zalio, 2003). Les relations sociales, repérées via la coprésence au sein des mêmes institutions (conseils d'administration de cercles bourgeois, clubs de sport huppés et principales entreprises et institutions économiques de Marseille), tissent un réseau qui contribue à la forte intégration sociale des élites patronales locales, et permettent de contrebalancer la concurrence intense entre les acteurs économiques de la région. 
Pour mieux comprendre les formes de solidarité limitée à l'œuvre dans la structuration des interlocks parmi les principaux administrateurs du CAC40, nous considérons ici les écoles fréquentées par les dirigeants, les grands corps de l'État, ainsi que les instances dirigeantes de think tanks marqués idéologiquement comme autant de cercles sociaux pouvant éclairer la structure des liens interlocks entre les sociétés du CAC40. On ne peut pas strictement parler de relation de cause à effet dans le deuxième cas : il est parfois difficile de déterminer si la coprésence dans les instances dirigeantes du think tank est antérieure à la formation du lien interlock. En l'absence d'éléments chronologiques probants, on se contentera de souligner le degré et le sens de la corrélation. Le fait que certains liens interlocks recoupent des liens de coappartenance à des think tanks montre toutefois la multiplexité des échanges (c'est-à-dire le fait qu'ils entretiennent des liens de différentes natures) entre les administrateurs concernés : leurs relations sociales dépassent le cadre de la gouvernance d'entreprise puisqu'elles ont, en l'occurrence, également trait à la défense d'idées politiques pour lesquelles ils se mobilisent activement.

\section{La méthodologie et les sources mobilisées}

La méthodologie consiste à comparer, au sein du réseau interlocks, la densité relative des sous-groupes formés par les administrateurs diplômés des mêmes écoles ou appartenant aux mêmes think tanks ou clubs, afin d'identifier des régularités dans la formation des interlocks suggérant l'existence d'un mécanisme de solidarité limitée ${ }^{5}$ entre administrateurs partageant certaines propriétés ou affiliations. Pour étudier un réseau interlocks, il est nécessaire de circonscrire au préalable une population d'entreprises. Nous avons retenu les 40 sociétés qui composent l'indice boursier CAC40 au 31 décembre 2006. Le décalage entre le nombre de sièges (776) et le nombre d'administrateurs (649) s'explique par le fait que certains dirigeants occupent simultanément des sièges dans plusieurs entreprises du corpus : 63 dirigeants sont présents dans 2 sociétés du CAC40, 19 dirigeants dans 3 sociétés, 6 dirigeants dans 4 sociétés et 2 dirigeants dans 5 sociétés. Nous avons choisi de nous intéresser aux 27 dirigeants présents dans au moins 3 sociétés du CAC40. Ces dirigeants, représentant moins de $5 \%$ de la population totale, forment une «élite de premier ordre » du point de vue des liens interlocks.

Reposant sur diverses sources disponibles, notre démarche ressemble à celle de l'historien lorsqu'il opère un travail prosopographique. La difficulté pour rencontrer et interviewer de manière systématique chacun des individus de la configuration étudiée motive en partie le mode de collecte de données. Parallèlement, ce qui rend possible une enquête «à distance », c'est la multiplicité des matériaux disponibles sur la population dans la mesure où sa position à la tête des plus grandes entreprises du pays s'accompagne d'une forte médiatisation. Une analyse à partir d'informations de seconde main issues de sources hétérogènes pose toutefois de multiples questions relatives aux biais de la méthode, aux modes d'objectivation des données et à ce que nous permettent de voir ou non les matériaux

\footnotetext{
5 «Cette solidarité limitée peut se mesurer de plusieurs manières, par exemple par une certaine stabilité dans la manière dont les membres choisissent leurs partenaires et définissent des intérêts communs à long terme, par l'existence de cycles caractérisant l'échange généralisé, par la présence de règles informelles interdisant de tirer la couverture à soi en matière de crédit ou imposant l'acceptation de la multiplexité. » (Lazega, 1999, p. 647)
} 
collectés. Notre analyse des liens interlocks s'appuie donc principalement sur trois types de matériaux : des listes, des notices et des récits biographiques.

La spécificité de notre article est d'utiliser des listes de coappartenances comme des données relationnelles, en nous fondant sur la dualité des groupes (Breiger, 1974) : le recours à de telles listes permet de transformer un matériau qualitatif mobilisable pour décrire des relations, en données quantitatives permettant de compter des liens. Si l'on est assuré de l'exhaustivité de certaines listes, comme celles des membres des instances dirigeantes des entreprises, il est parfois difficile d'obtenir ce type d'information lorsqu'il n'existe aucune contrainte juridique qui oblige les institutions à publier la liste de leurs membres ou dirigeants (think tanks, clubs et cercles mondains, etc.). En outre, la coappartenance à une même instance de gouvernance signifie automatiquement que les dirigeants se connaissent et se fréquentent plusieurs fois par an, mais ne nous livre pas d'informations quant au contenu réel de la relation (Stinchcombe, 1990). Un lien interlock n'exclut notamment pas l'existence d'antagonismes temporaires ou permanents entre des dirigeants. De plus, il est délicat à partir des listes de coappartenances d'orienter les liens ( $c f$. Encadré 2) : c'est pourquoi nous avons pris le parti de ne pas le faire.

\section{Encadré 2 : Un réseau interlocks non-orienté}

Orienter un réseau revient souvent à admettre que c'est le «numéro un » de l'entreprise qui invite les autres dirigeants dans son conseil d'administration. Or dans bien des cas, la situation est plus complexe, comme le montre la nomination en 2002 de Jean-René Fourtou comme PDG de Vivendi à la suite de Jean-Marie Messier: c'est Claude Bébéar, administrateur de Vivendi, qui est à l'initiative de l'éviction de J.-M. Messier de son conseil d'administration (Vuillermot, in Daumas, 2010, p. 70). De plus, l'orientation du réseau signifie également une perte d'information relationnelle. En effet, dans un réseau orienté, si A coopte $\mathrm{B}$ et $\mathrm{C}$, la relation entre $\mathrm{B}$ et $\mathrm{C}$ ne peut être orientée donc disparaît, alors qu'ils n'en sont pas moins reliés par un interlock. Or cette information peut être cruciale : il est, par exemple, peu probable que des dirigeants à la tête d'entreprises concurrentes se cooptent l'un l'autre car ces liens interlocks seraient considérés, à juste titre, comme collusifs. Il est néanmoins possible que ces mêmes dirigeants se côtoient au sein des conseils d'administration d'autres entreprises : à titre d'exemple, Michel Pébereau, président de la banque BNP Paribas, et Baudouin Prot, directeur général-administrateur de BNP Paribas, entretiennent chacun un lien interlock avec Daniel Bouton, PDG de la Société Générale, via respectivement les conseils d'administration des entreprises Total et Veolia Environnement. Ainsi, les interlocks, mêmes lorsqu'ils sont analysés comme la résultante de logiques individuelles, ne doivent pas nous faire oublier qu'ils lient des dirigeants à la tête d'organisations spécifiques. En résumé, la réduction de la qualité de l'information sociographique, résultat de l'introduction de postulats non présentées comme tels, pose la question des fondements mêmes de ce qui se trame derrière un lien interlock.

L'étude des notices contenues dans les annuaires biographiques permet à la fois de collecter des données sur les dirigeants (âge, sexe, nationalité, origine sociale, trajectoires scolaire et professionnelle) ainsi que sur leurs affiliations passées ou présentes. Le Who's Who in France constitue une de nos principales sources. L'annuaire présente cependant des 
incertitudes et des lacunes, largement liées à la manière dont sont produites les notices. Quel que soit l'annuaire considéré, il faut poser la question de sa fabrication, de sa fonction et du public qu'il vise, comme l'ont fait les travaux français sur les listes sociales à envergure nationale (Lewandowski, 1974 ; Grange, 1996) ou plus locale (par exemple, Duriez, 1990 ; Zalio, 2003). La sélection des personnalités dans le Who's Who se fait à l'initiative de l'éditeur, qui envoie à chacune un questionnaire de quelques pages (voir à ce sujet Lewandowski, 1974). Or, même si l'éditeur fait un travail de vérification, rien n'empêche un dirigeant de mettre en avant certaines périodes de sa vie, de laisser planer un doute sur un fragment de sa trajectoire professionnelle ou d'omettre volontairement son affiliation à certaines institutions $^{6}$. Il faut donc mobiliser avec précaution ce qui reste cependant une source importante de données sur les dirigeants ${ }^{7}$.

Les récits biographiques dans leur diversité constituent le troisième type de sources que nous mobilisons. Bien que le matériau soit difficile à objectiver, certains sociologues en font le cœur de leur analyse. Ces récits, souvent d'une grande valeur informative, recèlent de données concernant les profils, les pratiques, les fréquentations et les discours des dirigeants. On ne doit cependant pas écarter le caractère partial du matériau, cristallisé par les multiples « omissions », « déplacements », « inversions » et « condensations » des récits par rapport à la réalité des faits (Villette et Vuillermot, 2005, p. 69-passim), que nous révèle le travail de recoupement et de critique des sources.

\section{La configuration sociale de l'élite de premier ordre}

\section{Un petit monde}

Quelles sont les principales caractéristiques structurales des interlocks parmi les 27 dirigeants ? La Figure 1 est le graphe représentant ce réseau ${ }^{8}$. Il contient 114 liens, dont 17 correspondent à des liens multiples du fait de la coappartenance de deux dirigeants à plusieurs instances dirigeantes. Sa densité est très élevée : près d'un tiers des connexions possibles entre administrateurs sont effectives dans le réseau $(32,5 \%)^{9}$. Le réseau est constitué d'une seule composante connexe : chaque dirigeant est interconnecté, de manière plus ou moins directe, à

6 Nous pouvons évoquer ici deux exemples relatifs à la question de la neutralité du Who's Who. Certaines informations sont ambiguës : dans une notice, l'indication «ingénieur des Mines » est inscrite alors que le dirigeant n'est pas membre du grand corps technique le plus prestigieux (corps des Mines), mais diplômé de l'École des mines de Paris (ce qui fait de lui un ingénieur civil). Autre exemple, l'absence de mention de l'appartenance au club Le Siècle (attestée par une liste officielle, mais non publique) dans une autre notice : s'il est possible de l'interpréter comme un oubli, nous pensons qu'il existe une intention délibérée du dirigeant de ne pas faire figurer ce type d'informations.

Sans que cela résolve tous les problèmes, on peut croiser le contenu de notices issues d'annuaires différents et comparer les éditions successives d'un même annuaire (au fil des années, certaines informations changent ou disparaissent, d'autres apparaissent). La consultation des annuaires d'anciens élèves permet d'améliorer la qualité du matériau collecté. L'affection au sein des grands corps de l'État est publiée au Journal officiel. Quand on y a accès, on peut également retracer la carrière des dirigeants à partir des archives des entreprises qu'ils ont dirigées.

8 Le graphe a été généré avec le logiciel d'analyse de réseaux Pajek, au moyen de l'algorithme KamadaKawai.

9 La densité, comprise entre $0 \%$ (lorsque tous les individus sont isolés) et $100 \%$ (dans le cas d'une clique, lorsque tous les individus sont reliés entre eux deux à deux) est obtenue en divisant le nombre de liens observés dans le réseau par le nombre maximum de liens possibles. 
tous les autres. La distance géodésique moyenne est égale à 1,77 pas : un administrateur peut en moyenne atteindre n'importe quel autre membre du réseau directement (en un pas) ou indirectement (en deux pas ou plus). D'ailleurs, les administrateurs les plus éloignés dans le réseau ne sont séparés que par deux intermédiaires (trois pas).

\section{Figure 1. Liens interlocks entre les dirigeants de l'élite de premier ordre}

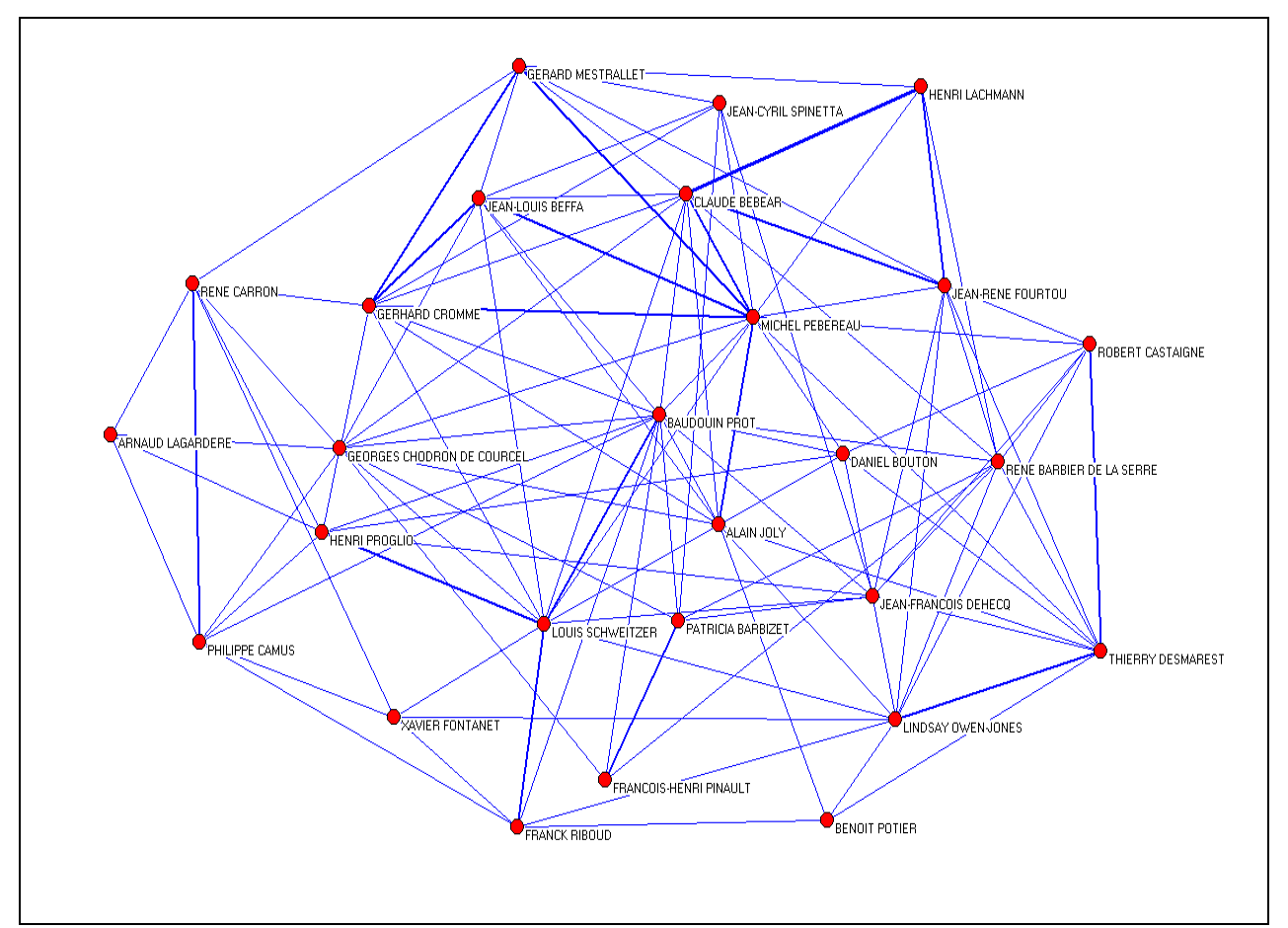

Lecture: Dans le graphe, les sommets représentent les administrateurs et les arêtes représentent les liens interlocks. Plus l'arête qui relie deux sommets est épaisse, plus il existe de liens interlocks entre les deux administrateurs.

Le réseau formé par les 27 membres de l'élite de premier ordre semble dense et cohésif, mais il est délicat à ce stade d'affirmer que sa topologie, en apparence singulière, est la résultante ou non de logiques sociales. Afin de le déterminer, nous comparons le réseau des interlocks à des réseaux aléatoires de manière à ne pas surinterpréter des propriétés qui relèveraient en fait de contraintes juridiques et organisationnelles. Nous avons généré 50 réseaux bimodaux ${ }^{10}$, construits de manière à posséder de nombreuses caractéristiques identiques à celles du réseau interlocks réel ${ }^{11}$.

10 Le terme «bimodal » qualifie un réseau composé de deux types de sommets différents, ici des sommets « entreprises » et des sommets « dirigeants ». A contrario, dans les réseaux unimodaux, tous les sommets sont de même nature.

11 Nous remercions Benoît Desmarchelier, doctorant en économie au Clersé, pour la programmation des réseaux aléatoires. L'algorithme décrit les contraintes suivantes : (a) chaque réseau bimodal est composé de 27 sommets «dirigeants» et de 40 sommets «entreprises » reliés par 91 liens dirigeants-entreprises; (b) les entreprises sont choisies par les dirigeants par un tirage dans une loi uniforme ; (c) chaque dirigeant est présent dans 3 à 5 conseils d'administration et chacune des 40 entreprises ne peut accueillir plus de 18 dirigeants. Enfin, chaque réseau bimodal est transformé en un réseau unimodal pour lequel nous avons calculé les indicateurs structuraux classiques. 
Les différences sont notables entre le réseau réel et les réseaux aléatoires (voir Tableau 1). La densité et la proportion de liens multiples du réseau sont plus grandes dans le réseau de l'élite de premier ordre que dans les réseaux aléatoires, tandis que le diamètre ainsi que la distance géodésique moyenne sont plus faibles. Ces différences montrent que la structure du réseau interlocks est effectivement le résultat de logiques sociales. Elles légitiment le fait de prendre en compte les attributs et affiliations des acteurs pour déterminer la nature de ces logiques.

Tableau 1. Comparaison entre le réseau interlocks réel et les 50 réseaux aléatoires

\begin{tabular}{|c|c|c|}
\hline & Réseau interlocks réel & Réseaux aléatoires* \\
\hline Densité unimodale & $32,5 \%$ & $\begin{array}{c}25,6 \% \\
(2,45 \%)\end{array}$ \\
\hline Nombre total de liens & 114 & $\begin{array}{c}89,9 \\
(8,60)\end{array}$ \\
\hline $\begin{array}{l}\text { Proportion de liens } \\
\text { multiples }\end{array}$ & $14,9 \%$ & $\begin{array}{c}11,7 \% \\
(4,18 \%)\end{array}$ \\
\hline Diamètre & 3 & $\begin{array}{c}3,67 \\
(0,59)\end{array}$ \\
\hline $\begin{array}{l}\text { Distance géodésique } \\
\text { moyenne }\end{array}$ & 1,77 & $\begin{array}{c}1,98 \\
(0,12)\end{array}$ \\
\hline
\end{tabular}

* Pour les réseaux aléatoires, les résultats donnés correspondent à une moyenne pour 50 réseaux. Les écarttypes sont entre parenthèses.

\section{La morphologie sociale de l'élite de premier ordre}

D'un point de vue socio-démographique, la configuration considérée semble extrêmement homogène. Les membres de l'élite de premier ordre forment un univers quasiexclusivement masculin (une femme sur les 27 dirigeants). Les membres de l'élite de premier ordre sont essentiellement de nationalité française: on ne compte que deux dirigeants étrangers, alors que la plupart des actionnaires déclarés des grandes entreprises françaises cotées à la bourse de Paris sont étrangers (Comet et Pizarro, 2011). La dispersion statistique des âges des dirigeants est relativement faible : la plupart d'entre eux sont nés entre la fin des années 1930 et le tout début des années 1950. Il est plus difficile d'obtenir des données systématiques concernant l'origine sociale des dirigeants. Nous avons réussi à recueillir 
suffisamment d'éléments d'information sur la profession du père pour positionner 20 des 27 administrateurs dans une hiérarchie sociale découpée en quatre catégories. Parmi eux, on recense 14 dirigeants issus de la haute bourgeoisie, 3 issus de la classe moyenne supérieure, 3 issus de la classe moyenne et aucun issu des classes populaires. L'étude des parcours scolaires des 27 dirigeants renforce encore l'impression d'homogénéité sociale.

Les membres de l'élite de premier ordre ont majoritairement fréquenté des grandes écoles, c'est-à-dire des «établissements d'enseignement supérieur qui recrutent leurs élèves par concours et assurent des formations de haut niveau » (JO du 11 septembre 1992). 24 des 27 dirigeants de la configuration (soit près de $90 \%$ de l'effectif total), ont étudié au sein d'une ou de plusieurs grandes écoles. En fait, les dirigeants ont fréquenté majoritairement quelques institutions, à savoir l'École polytechnique (Polytechnique ou X), l'École nationale d'administration (ENA), l'Institut d'études politiques de Paris (Sciences Po) et l'École des hautes études commerciales (HEC). Parmi les 24 dirigeants, on en dénombre 16 qui sont passés par au moins l'une de ces quatre institutions. Les 8 dirigeants restants ont fréquenté d'autres grandes écoles d'ingénieurs ou de commerce parisiennes. Michel Pébereau et Gérard Mestrallet, tous les deux diplômés de l'X et de l'ENA, sont les dirigeants les mieux dotés en capital scolaire. Or Michel Pébereau (Encadré 3) est aussi l'un des administrateurs qui cumule le plus de liens interlocks dans ce petit monde. Il est lié à 14 des 26 autres membres de la configuration sociale. Non seulement il occupe une position extrêmement centrale dans le réseau, mais il fait également partie de sous-groupes, où se joue une forte solidarité, comme en témoignent ses cinq liens interlocks doubles.

\section{Encadré 3 : Michel Pébereau ${ }^{12}$}

Né en 1942 dans une famille bourgeoise, Michel Pébereau est le fils d'un conservateur des hypothèques. Après une scolarité dans les lycées Buffon et Louis-le-Grand, il intègre l'École polytechnique, puis l'ENA. Sorti «dans la botte », il intègre le corps de l'Inspection des finances (IGF) en 1967 et entame une carrière de haut fonctionnaire au sein du ministère de l'Économie et des Finances, avant de pantoufler suite à l'arrivée de la gauche au pouvoir. Ayant rejoint le Crédit commercial de France, il participe à la privatisation de la banque en 1987 et en devient PDG jusqu'en 1993. Nommé à la tête de la BNP par le gouvernement Chirac, il supervise également sa privatisation. En 2003, Michel Pébereau cède la direction exécutive du groupe BNP Paribas à Baudouin Prot (HEC-ENA, IGF) et occupe depuis lors la présidence du conseil d'administration. Figure centrale du patronat français, il siège de manière durable, depuis les années 1990, dans les conseils d'administration de grandes sociétés françaises (Lafarge, Saint Gobain, Elf devenue Total, AXA) et, plus récemment, au sein de l'entreprise franco-allemande EADS (depuis 2007). En outre, il est membre du

\footnotetext{
12 Cette notice biographique (et la suivante, consacrée à Claude Bébéar, $c f$. Encadré 4) s'appuie principalement sur plusieurs éditions du Who's Who in France et du Bottin Mondain, des rapports annuels de sociétés, ainsi que sur des éléments biographiques contenus dans Villette et Vuillermot (2005), Daumas (2010) et Dudouet et Grémont (2010). De par leur position dans le réseau et leur influence effective au sein de l'univers du CAC40, les dirigeants qui font l'objet d'une notice biographique ne constituent pas des représentants typiques de la configuration sociale, mais plutôt deux pivots participant à la structuration du grand patronat français. En revanche, les caractéristiques socio-démographiques, trajectoires scolaires et carrières professionnelles de Michel Pébereau et Claude Bébéar sont relativement représentatives de celles des autres membres de l'élite de premier ordre.
} 
conseil exécutif du Medef et siège dans les instances dirigeantes de l'Institut Aspen France et de l'Institut de l'Entreprise.

\section{Formes de solidarité limitée}

\section{Les liens interlocks comme expression de solidarités scolaires?}

Le fait d'avoir fréquenté la même grande école augmente-t-il les chances d'être dans un même conseil d'administration ? Tel devrait être le cas s'il existe effectivement un esprit de corps entre les alumni des établissements les plus prestigieux (Bourdieu, 1989). Pour éclairer cette question, comparons, dans un premier temps, les densités des sous-réseaux formés par les dirigeants issus des mêmes grandes écoles ${ }^{13}$. Les quatre sous-réseaux composés d'au moins 3 dirigeants sont les sous-réseaux Polytechnique (8 dirigeants), ENA (6), Sciences Po (6) et HEC (4). Les calculs de densité indiquent que, dans le réseau interlocks, les polytechniciens et les énarques forment deux sous-réseaux beaucoup plus denses que le réseau général. A contrario, les anciens d'HEC n'entretiennent pas plus de liens entre eux qu'avec les autres dirigeants du réseau. Quant aux anciens de Sciences Po, ils semblent peu solidaires du point de vue des liens interlocks ${ }^{14}$. L'ensemble des résultats est consigné dans le Tableau 3.

Pour conforter ces résultats d'un point de vue statistique, nous avons généré - à l'aide d'une fonction du logiciel d'analyse de réseaux Ucinet $^{15}$ - un grand nombre de graphes aléatoires (plus de 1000 ) ayant la même densité globale que le réseau réel et des sous-réseaux aléatoires de même taille que les sous-réseaux réels. Les densités des sous-réseaux observés et des sous-réseaux attendus pour les graphes aléatoires sont ensuite comparées pour évaluer la probabilité qu'il n'y ait aucune corrélation avec l'appartenance à un cercle social particulier (par exemple, le cercle «Polytechnique »). Au final, on observe que, la plupart du temps, les sous-réseaux Polytechnique et ENA sont plus denses que leurs sous-réseaux aléatoires respectifs ${ }^{16}$.

Tableau 3. Homophilie scolaire des diplômés de l'X, l'ENA, Sciences Po et HEC

\begin{tabular}{|l|c|c|}
\hline & $\begin{array}{c}\text { Densité } \\
\text { du sous-réseau }\end{array}$ & $\begin{array}{c}\text { Centralité de degré moyenne } \\
\text { (dans l'ensemble du réseau) }\end{array}$ \\
\hline Réseau général $(N=27)$ & $32 \%$ & 8,4 \\
\hline Polytechnique $(N=8)$ & $64 \%$ & 10,0 \\
\hline
\end{tabular}

\footnotetext{
13 Il aurait été intéressant de disposer d'informations concernant les trajectoires scolaires antérieures au passage par les grandes écoles, car on sait que des amitiés durables peuvent se former dans les lycées et classes préparatoires, notamment les plus prestigieux (Bourdieu, 1989).

Ce qui prévaut dans les conseils d'administration n'est peut-être pas (ou moins) valable dans d'autres sphères sociales.

Fonction JointCount dans UCINET version 6.287 (Tools > Testing Hypotheses > Mixed Dyadic/Nodal $>$ Categorical Attributes > Joint-Count)

16 Dans $99,9 \%$ des cas pour Polytechnique et dans $98,4 \%$ pour l'ENA.
} 


\begin{tabular}{|l|l|l|}
\hline ENA $(N=6)$ & $60 \%$ & 9,7 \\
\hline Sciences Po $(N=6)$ & $20 \%$ & 8,7 \\
\hline HEC $(N=4)$ & $33 \%$ & 8,0 \\
\hline
\end{tabular}

Lecture: Les polytechniciens entretiennent deux fois plus de liens entre eux que les 27 dirigeants dans l'ensemble du réseau (densité de $64 \%$ contre $32 \%$ ). Ils ont néanmoins plus de liens interlocks que les autres d'une manière générale, ce qui pondère légèrement les écarts de densité intra-groupe : leur centralité moyenne (c'est-à-dire leur nombre de liens) est de 10 contre 8,4 pour l'ensemble des dirigeants.

L'analyse des liens multiples dans le réseau interlocks confirment ces résultats : alors que l'on dénombre 17 liens doubles sur l'ensemble des 114 liens du réseau (environ $15 \%$ ), plus d'un quart des liens entre polytechniciens sont multiples (5 liens sur 18); ils apparaissent dans les instances dirigeantes de sociétés présidées par des polytechniciens. La forte proportion de liens multiples et réciproques est un indice supplémentaire de l'existence d'une solidarité limitée entre polytechniciens. Les liens multiples sont moins fréquents dans le sousréseau ENA : seuls deux liens sur neuf sont doubles. Les polytechniciens et énarques ont en outre des centralités plus élevées que les autres. Dans la mesure où, d'après la littérature sur la diffusion, les leaders d'opinion ont généralement une forte centralité, cette position structurale traduit une capacité d'influence supérieure, pouvant se révéler très utile pour les entreprises. Le phénomène de solidarité limitée renforce le phénomène de sélection et induit une tendance à la centralisation du réseau.

Bien que difficile à étudier pour des raisons liées à la taille de notre corpus, il existe sans doute une structuration relationnelle du patronat selon les logiques de corps. L'anthropologue Irène Bellier notait à ce titre la volonté des élèves de l'ENA de renforcer leur cohésion pour contrebalancer le poids du corps des Mines (Bellier, 1992, p. 116). L'hypothèse de solidarité que nous faisons renvoie aux spécificités de l'X et de l'ENA, institutions scolaires desquelles est issue la «noblesse d'État», devenue «noblesse sans État » (Dudouet et Grémont, 2007). Cette hypothèse est difficilement vérifiable ici, du fait de la taille de la configuration. Notons néanmoins, d'une part, que les deux dirigeants X-Mines n'entretiennent pas de liens interlocks et, d'autre part, que le sous-réseau IGF $(N=4)$ forme une clique, mais que ses membres se rencontrent principalement au sein d'entreprises dont ils ne sont pas à la tête.

\section{Proximité idéologique, amitié et liens interlocks}

Nous nous intéressons maintenant aux coappartenances à un même groupement patronal, susceptibles de traduire des liens d'affinité idéologique voire des relations d'amitié parmi les dirigeants de l'élite de premier ordre. Les think tanks - ou cercles de réflexion constituent le premier type d'institution considéré. Après avoir dressé une liste (la plus exhaustive possible) des think tanks auxquels adhèrent les membres de l'élite de premier ordre, nous avons retenu les trois organisations ayant à leur tête au moins deux dirigeants de l'élite de premier ordre ${ }^{17}:$ l'Institut de l'entreprise, l'Institut Aspen France et l'Institut

17 Le faible nombre de think tanks fréquentés simultanément par les dirigeants de l'élite de premier ordre s'explique, au moins en partie, par la taille de la configuration et par le fait qu'il existe en France un grand nombre de cercles de réflexion. 
Montaigne. Ces trois cercles de réflexion ont pour point commun de défendre des valeurs libérales.

Les dirigeants membres de chacun des think tanks sont particulièrement interconnectés ${ }^{18}$. Le sous-réseau formé par les 3 dirigeants membres du bureau de l'Institut de l'entreprise est une clique. Celui formé par les quatre dirigeants membres du conseil d'orientation de l'Institut Aspen France est une composante connexe dont la densité est très élevée (83\%). De plus, trois des cinq liens qui unissent ces dirigeants sont doubles. Enfin, les deux dirigeants de l'Institut Montaigne sont liés par l'unique lien triple du réseau interlocks.

Deuxième constat, on observe que, parmi les dirigeants membres d'au moins un de ces think tanks, un seul n'est pas membre d'Entreprise et Cité en $2006^{19}$, un club de dirigeants libéral fondé en réaction à la victoire de la gauche aux élections présidentielles en 1981. Même si, selon Claude Bébéar, président d'Entreprise et Cité, le club est une association de dirigeants d'entreprise soucieux d'affirmer le rôle de l'entreprise dans la cité, ses membres la définissent plutôt comme une «bande de copains » qui se réunissent une fois par mois pour bavarder, pour partager de bons repas et pour aller chasser ${ }^{20}$. C'est dans le sens d'une relation entre des individus qui se fréquentent parce qu'ils s'apprécient et qui pensent pouvoir compter, dans une certaine mesure, les uns sur les autres que l'on parle ici de liens d'amitié entre dirigeants. Si la mesure de l'interconnexion entre des individus partageant des affiliations communes permet de repérer des réseaux denses et cohésifs, l'étude sociologique des liens interlocks passe aussi par un examen de la nature des relations considérées, laquelle nécessite le recueil et l'analyse de données qualitatives (entretiens, extraits d'interviews, témoignages, etc.).

À l'origine, Entreprise et Cité comptait une petite dizaine de dirigeants. Au cours des années 1990, le club patronal s'est élargi et comprenait environ vingt-cinq membres en 2006, tous dirigeants de très grandes entreprises et qui, comme le prétend C. Bébéar, n'auraient «jamais rien demandé à l'État ${ }^{21}$, alors que lui-même est issu de l'École polytechnique et que, parmi les membres, on dénombre plusieurs anciens hauts fonctionnaires ayant pantouflé, comme Michel Pébereau (IGF), Jean-Louis Beffa (Mines) et Gérard Mestrallet (direction du Trésor). En 2006, Claude Bébéar (voir Encadré 4), que la presse qualifie de «parrain » du patronat, fait partie des dirigeants les plus centraux dans le réseau (11 liens interlocks). En outre, il a trois liens multiples, dont l'unique interlock triple du réseau qui le lie à Henri Lachmann.

\section{Encadré 4 : Claude Bébéar}

Né en 1935, fils d'un directeur de collège et d'une institutrice, Claude Bébéar intègre les classes préparatoires du prestigieux Lycée Saint-Louis à Paris, avant d'entrer à l'École polytechnique. Son mauvais classement à la sortie de l'X ne lui permet pas d'intégrer un

\footnotetext{
18 Précisons que les cercles sociaux formés par les trois think tanks ne recoupent pas les cercles sociaux de type scolaire.

19 Il s'agit de Gerhard Cromme, PDG de ThyssenKrupp, qui est aussi le seul membre de l'élite de premier ordre ne résidant pas en France.

20 Jean Boissonnat, Nicolas Beau et Thierry Fabre, «Claude Bébéar, le baroudeur épicurien », L'Expansion, mai 1995.

21 Jean-Gabriel Fredet, «Capitalisme : les derniers 'parrains' », Le Nouvel Observateur, n $2108,2005$.
} 
grand corps. En 1958, il est recruté aux Anciennes mutuelles de Rouen, entreprise dont il prend très vite la tête. Multipliant les opérations d'acquisition et de fusion en France et à l'étranger, il transforme une société à rayonnement régional en un géant international de l'assurance : à la fin des années 1990, AXA est l'un des leaders mondiaux du marché de l'assurance. Claude Bébéar, qui exercera un pouvoir incontesté sur la société pendant plus de trente ans, se définit comme un patron attaché à la liberté d'entreprendre et se déclare volontiers éloigné de ce qu'il considère comme l'idéal de la plupart des polytechniciens et énarques, désireux de servir l'État, et conservant d'après lui une «mentalité de fonctionnaires », même lorsqu'ils pantouflent dans le privé. L'influence du dirigeant ne s'arrête pas aux frontières de la société qu'il dirige. À l'origine du club Entreprise et Cité (1983), fondateur de l'Institut du Mécénat humanitaire (1986) et de l'Institut Montaigne (2000), le patron d'AXA fait également partie, dès le début des années 1990, des organes de gouvernance de plus grandes sociétés françaises. En 2000, Claude Bébéar cède sa place de président du directoire d'AXA au profit d'Henri de Castries (HEC-ENA, corps de l'IGF) en échange de la présidence du conseil de surveillance.

Le sous-réseau interlocks «Entreprise et Cité » composé de 6 des 27 dirigeants du réseau général présente une structure singulière. Premièrement, on observe que sa densité est très élevée $(87 \%)$. Comme précédemment, à l'aide du logiciel UCINET, nous comparons nos résultats à ceux obtenus dans des sous-réseaux aléatoires. Il en résulte que la densité du sousréseau «Entreprise et Cité » est toujours supérieure à celles des sous-réseaux aléatoires de même taille. En outre, dans le sous-réseau réel, on observe que près de $50 \%$ des liens sont multiples (contre $15 \%$ dans le réseau général). On note enfin que l'ensemble des liens interlocks entre les six dirigeants du club Entreprise et Cité résulte de la coprésence dans cinq conseils d'administration de sociétés du CAC40, toutes dirigées par l'un des six membres du club patronal, ce qui dénote une forte réciprocité en matière de cooptation.

Au sein du réseau considéré, la formation des liens interlocks renvoie donc à une forme de solidarité limitée, reposant sur des bases idéologiques et affinitaires : les dirigeants membres de l'élite de premier ordre, qui se côtoient dans certains espaces de sociabilité marqués idéologiquement (ici, des think tanks libéraux), ont tendance à se coopter dans les conseils des sociétés du CAC40 alors même qu'ils sont diplômés d'écoles différentes (X, ENA, HEC et formation universitaire pour l'un d'entre eux) et ont accompli des parcours professionnels différents (certains sont issus de la noblesse d'État, d'autres ont fait toute leur carrière dans le privé). La mise au jour de cette forme de solidarité limitée vient ainsi compléter les observations précédentes relatives à l'homophilie scolaire, qui a peut-être tendance à réifier l'effet structurant des passages par les grandes écoles, au détriment d'autres facteurs comme la proximité idéologique, étudiée à travers le prisme des think tanks, et des liens de camaraderie ou d'amitié plus difficiles à repérer et à objectiver.

\section{Conclusion}

Le principal intérêt de ces analyses est de démontrer l'existence de formes de solidarité limitée parmi les administrateurs du CAC40. L'analyse de la solidarité scolaire éclaire la persistance d'une caste de dirigeants, issus de la noblesse d'État, qui se cooptent 
dans les conseils d'administration. Le réseau interlocks des patrons du CAC40 - présidents du conseil et DG - est toujours dominé en 2007 par les dirigeants issus de la haute administration, même s'ils sont devenus relativement autonomes vis-à-vis de la tutelle étatique (Dudouet et Grémont, 2007) : les patrons «corpsards » contrôlent les plus grosses sociétés et sont particulièrement centraux dans le réseau interlocks. Par ailleurs, nous montrons que la solidarité limitée repose aussi sur des bases idéologiques et affinitaires : certaines coappartenances dans les conseils d'administration coïncident avec des coappartenances dans des think tanks libéraux et un club patronal. Ce résultat renvoie à l'existence d'un Inner Circle (Useem, 1984), composé de dirigeants, fortement interconnectés, mobilisés pour défendre des valeurs libérales en vue d'influencer l'agenda politique.

La mise au jour de ces formes de solidarité limitée permet de mieux comprendre le paradoxe français lié à la stabilité de l'élite patronale dans un contexte de profond changement institutionnel, avec notamment l'internationalisation des grandes sociétés et la montée des investisseurs étrangers dans leur capital. Notre analyse porte toutefois sur les administrateurs cumulant le plus de sièges dans les conseils d'administration du CAC40 fin 2006. Depuis lors, la morphologie du réseau formé par ces mêmes administrateurs a changé. En 2010, le réseau interlocks des 27 n'est plus connexe : deux dirigeants (Gerhard Cromme et René Barbier de la Serre) sont isolés du composant principal. La densité $(17,3 \%)$ a été divisée par deux en quatre ans et le nombre de liens multiples par plus de trois (5 contre 17). Cette évolution est en partie due à une logique démographique de renouvellement des élites dirigeantes et à l'effet de la limite d'âge pour l'exercice de certains mandats, mais reflète également une tendance plus générale de diminution des liens interlocks, que l'on mesure dans de nombreux autres pays industrialisés.

Par ailleurs, nous avons mis l'accent sur l'effet de la solidarité limitée parmi certains administrateurs, en mettant de côté les interdépendances entre les sociétés. Or les facteurs organisationnels ont bien entendu un poids considérable. Notons à ce propos que la plupart des administrateurs les plus centraux dans le réseau étudié relèvent du cœur financier du capitalisme français. Pour dépasser l'opposition entre logiques organisationnelles (les dirigeants sont au service des firmes) et individuelles (les entreprises sont instrumentalisées par les dirigeants), il faudrait mobiliser des modèles explicatifs qui intègrent les deux types de facteurs, qui ne sont pas incompatibles et doivent certainement parfois se superposer. Des liens interlocks peuvent ainsi se constituer préalablement à une fusion ou à une acquisition et recouper des liens sociaux entre les dirigeants de chacune des sociétés, se connaissant de longue date et s'appréciant l'un l'autre. Gageons par ailleurs que l'introduction d'autres cercles sociaux - par exemple, ceux liés à la parenté, à la religion, aux trajectoires scolaires antérieures aux grandes écoles, etc. - permettrait d'approfondir le mécanisme de solidarité limitée à l'œuvre dans les jeux d'intercooptation.

Enfin, si notre méthodologie permet d'objectiver à partir de régularités statistiques des facteurs difficiles à démêler avec des approches uniquement qualitatives ${ }^{22}$, il est difficile de

\footnotetext{
22 En effet, les processus de nomination peuvent être complexes, inclure de nombreux acteurs et s'étaler dans le temps. A partir d'entretiens, il n'est pas toujours possible de reconstituer de manière robuste tous les échanges, jeux d'influence et compromis qui ont eu lieu et encore moins de savoir précisément quels sont les facteurs qui ont été les plus déterminants.
} 
mesurer la part relative de chacun d'entre eux. Néanmoins, que les liens interlocks recoupent des affiliations aux mêmes grandes écoles ou aux mêmes think tanks, ils évoquent dans les deux cas l'existence de liens d'interconnaissance et d'inter-reconnaissance (Bourdieu, 1980). Nous avons précédemment montré, à partir d'une analyse portant sur les patrons des 100 sociétés les plus capitalisées cotées à la bourse de Paris, que l'effet des diplômes tend d'ailleurs à s'estomper, dans des modèles multi-variés, au profit de l'origine sociale (Comet et Finez, 2010). La principale limite de notre méthodologie tient plutôt à la question de l'interprétation des liens interlocks : quel sens leur confèrent les acteurs ? De ce point de vue, cette méthodologie gagnerait à être couplée à des entretiens semi-directifs auprès de l'ensemble de la population enquêtée. 


\section{Bibliographie}

BAUER (M.), 1988. Grands patrons, capital, État et entreprise, «les 200 » révèlent, Sociologie du travail, vol. 30, $\mathrm{n}^{\mathrm{o}} 4$, p. 567-584.

BAUER (M.), BERTIN-MOUROT (B.), 1987. Les 200. Comment devient-on un grand patron?, Paris : Seuil.

BELLIER (I.), 1992. Regard d'une ethnologue sur les énarques, L'Homme, vol. 32, nº 121, p. 103-127.

BOURDIEU (P.), 1980. Le capital social. Notes provisoires, Actes de la recherche en sciences sociales, $\mathrm{n}^{\mathrm{o}} 31, \mathrm{p} .2-3$.

BOURDIEU (P.), 1989. La noblesse d'État. Grandes écoles et esprit de corps, Paris: Éditions de Minuit.

BOURDIEU (P.), de SAINT MARTIN (M.), 1978. Le Patronat, Actes de la recherche en sciences sociales, $\mathrm{n}^{\mathrm{0}}$ 20-21, p. 3-82.

BREIGER (R.L.), 1974. The duality of persons and groups, Social forces, vol. 53, $\mathrm{n}^{\circ} 2$, p. 181-190.

COMET (C.), FINEZ (J.), 2010. Le cœur de l'élite patronale, Sociologies Pratiques, $\mathrm{n}^{\circ} 21$, p. 49-66.

COMET (C.), PIZARRO (N.), 2011. The Cohesion of Intercorporate Networks in France, Procedia Social and Behavioral Sciences, vol. 10, p. 52-61.

DAUMAS (J.-C.) (dir.), 2010. Dictionnaire historique des patrons français, Paris : Flammarion.

DUDOUET (F.-X.), GREMONT (E.), 2007. Les grands patrons et l'État en France : 19812007, Sociétés contemporaines, $\mathrm{n}^{\mathrm{o}}$ 68, p. 105-131.

DURIEZ (B.), 1990. La bourgeoisie répertoriée : le Livre des Familles du Nord, Ethnologie française, vol. $20, \mathrm{n}^{\mathrm{o}} 1$, p. 71-84.

FINEZ (J.), 2009. La solidarité des dirigeants du CAC 40. Une analyse des liens interlocks entre les membres de l'élite de premier ordre, Mémoire de Master 2, s. dir. Catherine Comet, Univ. Lille 1.

GRANGE (C.), 1996. Les gens du Bottin Mondain. Y être, c'est en être, Paris : Fayard.

GRANOVETTER (M.S.), 1995. The economic sociology of firms and entrepreneurs, in A. Portes (ed), The economic sociology of Immigration, New York : Russel Sage.

KADUSHIN (C.), 1995. Friendship among the French Financial Elite, American Sociological Review, vol. 60, $\mathrm{n}^{\mathrm{o}} 2$, p. 202-221.

LAZEGA (E.), 1999. Le phénomène collégial : une théorie structurale de l'action collective entre pairs, Revue française de sociologie, vol. 40, $\mathrm{n}^{\circ}$ 4, p. 639-670.

LEWANDOWSKI (O.), 1974. Différentiation et mécanismes d'intégration de la classe 
dirigeante. L'image sociale de l'élite d'après le Who's Who in France, Revue française de sociologie, vol. $15, \mathrm{n}^{\mathrm{o}} 1, \mathrm{p} .43-73$.

MIZRUCHI (M.S.), 1996. What Do Interlocks Do: An Analysis, Critique, and Assessment of Research on Interlocking Directorates, Annual review of sociology, vol. 22, p. 271-298.

STINCHCOMBE (A.L.), 1990. Weak structural data : Review of "Intercorporate Relations: The Structural Analysis of Business. by Mark S. Mizruchi; Michael Schwartz”, Contemporary Sociology, vol. 19, n ${ }^{\mathrm{o}} 3$, p. 380-382.

SULEIMAN (E.N.), 1979. Les élites en France. Grands corps et grandes écoles, Paris : Seuil.

USEEM (M.), 1984. The Inner Circle. Large Corporations and the Rise of Business Political Activity in the U.S. and the U.K., New York: Oxford University Press.

VILLETTE (M.), VUILLERMOT (C.), 2005. Portrait de l'homme d'affaires en prédateur, Paris : La Découverte.

ZALIO (P.-P.), 2003. Un monde patronal régional dans les années 1930. Une perspective configurationnelle, Cahiers lillois d'économie et de sociologie, $\mathrm{n}^{\mathrm{0}}$ 41-42, p. 137-176. 